\title{
Mobile Network Competition, Customer Ignorance and Fixed-to-Mobile Call Prices
}

\author{
by
Joshua S. Gans and Stephen P. King* University of Melbourne

First Draft: $19^{\text {th }}$ December, 1999

This Version: 14th February, 2000

\begin{abstract}
This paper examines the influence of mobile network competition on the prices of fixed-to-mobile calls. Because fixed line customers cannot, in general, distinguish the identity of a specific mobile network, these networks have market power when setting termination charges for calls from fixed lines. We show that: (1) unregulated mobile termination charges will result in higher than monopoly call prices; (2) the regulation of termination charges and prices downward will affect mobile subscription rates and may lower these rates; and (3) regulation of any mobile carrier's termination charges can reduce fixed to mobile prices but will result in an increase in unregulated carriers' termination charges. When fixed line consumers can distinguish between the different mobile networks they are calling, fixed to mobile call prices will fall relative to their level under customer ignorance. Direct mobile charging for termination also exerts downward pressure on the total fixed to mobile call price. A low cost method of lowering fixed to mobile charges would be to facilitate the identification of carriers by consumers and to restructure billing so that mobile networks are able to directly charge fixed line consumers for termination services. Journal of Economic Literature Classification Numbers: L41, L96.
\end{abstract}

Keywords. Mobile network competition, termination charges, interconnect fees, price regulation and customer ignorance.

\footnotetext{
* We thank the Australian Competition and Consumer Commission (ACCC) for providing funds for this project. The views expressed are solely those of the authors and do not reflect those of the ACCC. All correspondence to: Joshua Gans; E-mail: J.Gans@mbs.unimelb.edu.au. The latest version of this paper can be found at http://www.mbs.unimelb.edu.au/jgans.
} 


\section{Introduction}

Compared to other areas of telecommunications, mobile networks are in their infancy and, to date, there has been relatively little analysis of mobile network pricing. Recently, however, both UK and Australian competition authorities have become interested in the prices that mobile networks charge to other networks - in particular, fixed line networks that have an established customer base and coverage.

In December 1998, the UK Monopolies and Mergers Commission and the telecommunications regulator, OFTEL, released a report into the prices that the two largest mobile networks, Cellnet and Vodafone, charge for termination of fixed line calls made to their networks. They concluded that there was "currently insufficient competitive constraint on termination charges" (p.3) and recommended that those charges be regulated on the basis of cost. The Australian Competition and Consumer Commission (ACCC) is currently arbitrating several disputes between fixed line and mobile networks involving mobile termination charges. This follows the 'declaration' of GSM network terminating services by the ACCC. Declaration compels GSM networks to negotiate interconnection arrangements with other carriers, where the ACCC is the default arbitrator of any disputes. ${ }^{1}$ The ACCC has also expressed concern that competition between mobile networks may not lead to efficient fixed-tomobile termination charges (ACCC, 1999).

Recent research, such as Armstrong (1998), Laffont, Rey and Tirole (1998a, 1998b) and Carter and Wright (1999), has focussed on interconnection charges between competing telecommunications networks. But there has been little research into the setting of termination charges between networks based on different modes of telecommunications. With fixed-to-mobile pricing, the termination charges that are set independently by competing mobile networks influence the prices set by a noncompeting network; the fixed line carrier. As termination charges influence the value to a mobile network of attracting a customer, they play an important role in mobile competition. As we show, however, this competition may adversely affect fixed-line customers.

\footnotetext{
${ }^{1}$ King and Maddock (1999) analyse the Australian declaration and arbitration access regime.
} 
The competitive effects of mobile termination charges are influenced by what we call customer ignorance. By convention, in many countries (an exception is the US for calls to mobiles) the caller pays for making a call. However, fixed-line customers often cannot identify the specific mobile network that they are calling. They might wish to call a certain person or class of people who have mobile phone numbers, but they will often not be able to determine which mobile carrier is associated with each person or number. Even if fixed line networks take differentials in mobile network termination charges into account when setting fixed-to-mobile prices, the inability of fixed-line customers to identify mobile networks and, consequently, any price differentials in calls to them, means that fixed line customers are likely to base their fixed-to-mobile calling patterns on average prices. Alternatively, the fixed line carrier will set its price for fixed-to-mobile calls on the basis of average termination charges. ${ }^{2}$ These averages depend on the observed market shares of mobile networks. As a result, an increase in one mobile network's termination charges raises the average price of fixed-to-mobile calls and lowers demand for all of these calls. There is a horizontal externality imposed on the termination revenues of other mobile networks as well as a vertical externality imposed on the fixed line networks. ${ }^{3}$ In this paper we find that both of these externalities are potential sources of inefficient pricing.

Number portability exacerbates customer ignorance. Even where a prefix may initially provide some information about the network being called, with mobile number portability those differences will diminish over time, making mobile networks indistinguishable to fixed-line customers.

To see the effect of customer ignorance in more detail, suppose that the opposite is true and the A-party customer calling fixed-to-mobile both knows the identity of the terminating carrier and the price of the call. In some circumstances, a mobile network will retain some market power. If the A-party has to contact a specific person then they will still make the call, although if the per minute termination charge is high, they might truncate the call or ask the person on the mobile phone to call them

\footnotetext{
2 In its inquiry into mobile termination, the UK Monopolies and Mergers Commission found that fixed line consumers had little knowledge of the mobile networks they were calling or of price differentials in carrier-specific call prices; see MMC (1998, pp.31-33).

3 The vertical externality, which may lead to 'double marginalisation', has been thoroughly discussed in the literature. For example, see Perry (1989). The novel issue focused on in this paper is the horizontal externality created by customer ignorance.
} 
back. In other situations, the mobile carrier will have little market power. If the Aparty does not need to call a specific person, but rather can choose any individual from a group of people, then they will choose the individual who is cheapest to contact. For example, if the A-party needs to call a plumber, but has no preference over which plumber they contact, then they will choose the plumber that is linked to the mobile network with the lowest call charges. This, in turn, will make the plumber indirectly face the termination costs - if they join a mobile network with high termination charges then they will receive fewer calls and less business. A mobile network with higher termination charges will have fewer subscribers and competition will tend to moderate termination charges.

In contrast, suppose that the person making the fixed-to-mobile call is only able to guess at the identity of the terminating network. In particular, suppose that the A-party only knows the market shares of mobile carriers and can only estimate the average termination charge. Then each mobile network does not bear the full competitive consequences from raising their termination charges and, as a result, will have considerable discretion to raise these charges. When one network raises its termination charges, this raises the average price that the A-party pays. But the Aparty only knows this average and because they cannot distinguish between mobile networks, they will make their calling decisions on the basis of the average price, not the network specific charges. This, in turn, breaks the indirect link between termination charges and call frequency to a specific mobile customer.

Take the plumber example presented above. If the A-party cannot distinguish the identity of the terminating network before they make their call, then this identity is irrelevant to the decision about which plumber to call. The person may call a plumber on a network with high or low termination charges, but they are only likely to know this when they receive a bill. By then, it is too late for the A-party to change their calling decision. The plumber on a network that has high termination charges is no longer penalised through fewer calls for these charges, and so does not even indirectly bear these charges. In fact, to the degree that a network might pass some of these high termination charges back to a customer through lower prices for calls originating on the mobile network, the plumber might have an economic incentive to join a network with high termination charges.

High mobile termination charges will adversely affect fixed-line carriers' profits. This may increase the incentive for vertical integration. By integrating with a 
mobile network, the fixed-line network can potentially internalise the vertical externality.

High mobile termination charges will also raise regulatory concerns. In particular, should the authorities respond by regulating mobile termination charges or are there structural changes or administrative rules that can moderate regulatory concerns?

In this paper we present a model of customer ignorance and show how this affects mobile termination and call pricing when there are multiple mobile networks but a single fixed-line carrier. Our model is developed in two stages. First, in section 2, we consider a situation where the mobile networks are not close substitutes and their market shares are effectively fixed. This allows us to consider the horizontal and vertical effects of termination charging without considering their impact on subscriber competition between mobile networks. In section 3 we then examine what happens when two mobile networks compete against one another. We model this by assuming that termination charges are set prior to competition for customers by mobile networks. This extension to our model allows us to analyse the relationship between mobile termination charges and subscriber charges.

Our basic pricing results follow directly from the horizontal and vertical externalities noted above. Unregulated mobile termination charges will be higher than the monopoly pricing of such services. An increase in termination charges by one mobile network raises the average price for fixed-to-mobile calls and reduces both the fixed carrier's profits and, due to customer ignorance, the profits received by other mobile carriers. As a result, each mobile network has an incentive to overprice termination from both a social and an industry perspective. Indeed, the smaller (in terms of network share) a mobile network, the less likely is it to internalise the demand-reducing effects of an increase in its termination charge. So the less concentrated the mobile network market, the higher will be the level of fixed to mobile call charges.

The overpricing of termination is exacerbated by competition between mobile networks for subscribers. By having a higher termination charge, a mobile network effectively receives greater benefits from attracting an additional subscriber and, hence, can afford to offer more attractive subscription terms to that customer. In essence, termination revenues 'cross subsidise' subscriptions. In the extreme, mobile network competition could result in termination charges that 'choke-off' fixed to 
mobile demand entirely. That is, in equilibrium, mobile termination charges may be so high that the fixed carrier is unable to profitably offer fixed to mobile service. ${ }^{4}$

The fixed carrier might try and moderate high termination charges by integrating with one of the mobile carriers. If mobile carriers compete for the same group of customers, then integration, together with a refusal to deal with any nonintegrated mobile network, can result in a fixed and mobile monopoly. This would internalise the horizontal and vertical externalities but destroy any mobile competition. Alternatively, if mobile carriers effectively supply different groups of subscribers, then integration is of little benefit. Integration between the fixed carrier and one mobile carrier will reduce the average effective termination charge, as the integrated firm sets its implicit termination charge equal to marginal cost. But other non-integrated carriers will respond by raising their termination charges. We show that, as a result, the integrated carrier receives lower profits than if it was vertically separated and integration is not advantageous to the relevant networks.

Regulation might be used to moderate the competitive concerns about mobile termination charges. There are a variety of alternatives. ${ }^{5}$ For example, termination charges could be directly regulated. This is likely to result in lower termination charges, lower fixed-to-mobile prices and a greater intensity of fixed-to-mobile calls. But reducing termination charges will also affect the price of other mobile services, such as mobile subscriber charges. Naively, we might expect lower termination charges to result in less subscriber competition and higher subscriber charges. We show however that this is incorrect in the context of our model. Lower regulated termination fees can lead to higher termination revenues for the mobile carriers. These revenues then generate greater competition for mobile subscribers and lower charges to join a mobile network. Regulation can benefit both the fixed-line and the mobile customers.

\footnotetext{
${ }^{4}$ Note that overpricing of termination will occur even if mobile subscribers prefer more incoming calls as well as outgoing calls. So long as consumers on the fixed network cannot distinguish between alternative mobile networks when making calls, their demand will be based on an average price. If one mobile network reduces its termination charges then it may reduce the average price of fixed-to-mobile calls and increase the total number and duration of these calls, but it will not increase the relative number of calls its subscribers receive. As such, mobile networks have little ability to use differences in termination charges to attract consumers to their network. While a consumer preference for incoming calls may increase the attractiveness of subscribing to some mobile phone network, so long as there is customer ignorance, this will not exert significant competitive pressure on termination charges.

${ }^{5}$ One option that we have not addressed is to provide mobile consumers with a 'receiver pays' option for fixed to mobile calls, as is practiced in the United States. Recent research suggests that this may have a beneficial effect on fixed to mobile prices and mobile competition. See Doyle and Smith (1998).
} 
Rather than regulate all termination charges, authorities might only regulate termination for those mobile carriers with the greatest market share. However, as noted above for integration, selective reduction in some carriers' termination charges will result in an increase in other carriers' termination charges. ${ }^{6}$

We also consider other, more structural, regulatory procedures. For example, authorities may try to reduce customer ignorance by requiring mobile carriers to 'identify' themselves before call charging commences. We find that if consumers can distinguish between networks then termination charges are reduced, although they may still result in fixed-to-mobile call prices above monopoly levels due to double marginalisation.

One source of market power for mobile carriers is that termination charges are likely to be more difficult to change than the actual price of fixed-to-mobile calls and are embedded in these call prices. For example, changing termination charges may involve considerable inter-carrier negotiation whereas an unregulated fixed carrier will often be able to alter the price of fixed-to-mobile calls as it desires. This relative inflexibility allows the mobile carriers to act as price leaders in the setting of fixed-tomobile charges. A regulatory authority might improve competition by separating termination charges. This could be done, say, by separating a fixed line bill into two components - a terminating charge and a trunk-originating charge. In return for a regulated billing fee, the fixed line carrier would still do the actual billing and the caller would still pay for the calls. We show that this simple regulatory option would moderate the vertical externality between mobile and fixed carriers, resulting in lower fixed-to-mobile call prices. ${ }^{7}$

Overall, our analysis shows that mobile termination charges are unlikely to reflect termination costs and will tend to increase, rather than decrease, with mobile network competition. Regulation can moderate this problem, but authorities need to be aware that changes to termination charges may also effect mobile subscriber fees. Some arms-length regulatory procedures, such as carrier identification and direct mobile charging, can be used to reduce termination charges. However, reduction of

\footnotetext{
6 Gans and King (1999) consider the regulation of termination charges for 'non-dominant' mobile networks in detail.

7 Australia is currently pursuing the option of allowing fixed-line customers to pre-select the firm that will bill them for fixed-to-mobile calls. The pre-selected firm will negotiate directly with the fixed and mobile carriers regarding the origination, trunk and termination charges for fixed-to-mobile calls. If there is strong competition
} 
mobile termination charges to reflect termination costs is likely to require direct price intervention.

\section{Exogenous Mobile Network Market Shares}

We begin by analysing fixed to mobile call charges when mobile networks' market shares are exogenous. This simplifies the strategic interactions between networks and allows us to focus on the key determinants of termination charges. The exogenous market share case is most applicable to situations where mobile networks are not strong substitutes, for example if different networks cover different regions or consumer groups. This case abstracts from the effect of termination charges on competition between networks for subscribers. Consequently, in section 3, we extend the model to allow for mobile network competition and explore the interactions between fixed to mobile prices and other prices that may be set by fixed and mobile carriers. We will see that the logic of many of the results presented in this section continues to be valid even in this more complex framework. ${ }^{8}$

\subsection{Model Set-Up and Assumptions}

Consider a model with $n$ independent mobile networks and a single fixed network. Mobile phone customers are exogenously distributed between mobile networks where $s_{i}$ is the market share of mobile network $i$. The marginal cost of terminating a call on a mobile network is given by $c_{T}^{M}$ while the marginal cost of originating a call on the fixed network is $c_{O}^{F}$. The marginal cost of trunk services is given by $c_{1}$ so that the total marginal cost of a fixed-to-mobile call is given by $c_{F M}=c_{O}^{F}+c_{1}+c_{T}^{M}$.

Let $P_{i}$ be the price of a call from the fixed network to mobile network $i$. We can think of this price as either being a price per call or a price per minute of the call.

between pre-selected firms then the negotiated charges will be passed on directly to the customer and pre-selection will be identical to the direct charging option discussed here.

8 We could view the fixed share case as modelling a situation where mobile networks cannot commit to termination charges prior to signing-up subscribers. The analysis here would then reflect the interaction in the 
The fixed carrier sets this price. We assume that either (1) the fixed carrier is unable to set a different price for different mobile networks, so that $P_{i}=P_{j}=P$ for all mobile carriers or (2) that the customers cannot determine which mobile carrier they are ringing ex ante. Under this later assumption, the fixed carrier could set different prices for different mobile carriers but these prices would only influence consumers' decisions to the extent that they change the average price, $P$ that customers pay. This average price determines demand and is given by $P=\sum_{i} s_{i} P_{i}$. The second assumption captures the idea of customer ignorance discussed above.

As consumers either explicitly or effectively face a single (average) fixed-tomobile price, $P$, the demand for fixed-to-mobile calls is given by $Q(P)$. It is often convenient to assume that this demand is linear so that $Q=\frac{1}{2 b}(a-P)$. The linear demand assumption allows us to explicitly calculate prices and charges and to compare these charges over different regimes. The fixed carrier will set the price of fixed-to-mobile calls taking into account both the demand for such calls and the cost of those calls.

If the fixed and mobile networks are owned by separate firms, then the marginal termination cost that will enter the fixed carrier's pricing policy is not the true marginal cost of termination, $c_{T}^{M}$ but rather the marginal termination charge set by the relevant mobile carrier. We denote the marginal termination charge set by mobile carrier $i$ by $T_{i}$ per call.

A useful benchmark price for our analysis is the uniform monopoly price for fixed-to-mobile calls. This is the profit maximising price that would be set by a single firm that owned both the fixed network and the only mobile network. We denote this price by $P^{m}$, where it is implicitly defined for a general demand function by $Q^{\prime}\left(P^{m}\right)\left(P-c_{F M}\right)+Q\left(P^{m}\right)=0$. For the case of a linear demand, $P^{m}=\frac{1}{2}\left(a+c_{F M}\right)$ and the associated monopoly quantity is $Q^{m}=\frac{1}{4 b}\left(a-c_{F M}\right)$. Monopoly profits from the sale of fixed-to-mobile call services in this situation is denoted by $\Pi^{m}=\frac{1}{8 b}\left(a-c_{F M}\right)^{2}$ 


\subsection{Unregulated Outcomes Without Integration}

Suppose that the $n$ mobile networks independently and simultaneously set their linear termination charges $T_{i}$. The fixed network owner then sets the fixed-tomobile price $P$. Given this (average) price, customers decide how many fixed-tomobile calls they will make, taking into account their own ignorance of the identity of the terminating mobile carrier for any specific call. With each decision, the relevant firm seeks to maximise its profits and all networks take the market shares of each mobile carrier as given. Given the termination charges, the fixed carrier will set $P$ to solve:

$$
\max _{P}\left(P-c_{O}^{F}-c_{1}-\sum_{i} s_{i} T_{i}\right) Q(P)
$$

With linear demand, the solution to this problem is given by $P=\frac{1}{2}\left(a+\sum_{i} s_{i} T_{i}+c_{O}^{F}+c_{1}\right)$ and $Q=\frac{1}{4 b}\left(a-\sum_{i} s_{i} T_{i}-c_{O}^{F}-c_{1}\right)$. Given the behaviour of the fixed carrier, the mobile networks will simultaneously set their termination charges to solve:

$$
\max _{T_{i}} \frac{s_{i}}{4 b}\left(T_{i}-c_{T}^{M}\right)\left(a-\sum_{j} s_{j} T_{j}-c_{O}^{F}-c_{1}\right)
$$

The first order condition for the mobile carrier's optimisation problem is given by:

$$
a-\sum_{j \neq i} s_{j} T_{j}-2 s_{i} T_{i}-c_{O}^{F}-c_{1}+s_{i} c_{T}^{M}=0
$$

Solving these first order conditions simultaneously for all mobile carriers gives the Nash equilibrium termination charges.

There are three cases worth more detailed consideration. First, suppose that there is just one mobile carrier. This allows us to isolate the effects of vertical separation. In this situation, $s_{i}=1$, so that $T=\frac{1}{2}\left(a-c_{O}^{F}-c_{1}+c_{T}^{M}\right)$ and $P=\frac{1}{4}\left(3 a+c_{F M}\right)>P^{m}$ so long as $a>c_{F M}$. The total quantity of fixed to mobile calls is given by $Q=\frac{1}{8 b}\left(a-c_{F M}\right)$. This is the standard double marginalisation result - vertical separation of the fixed and mobile carriers leads to pricing above the monopoly level. This is detrimental for both consumers and for the firms themselves. The monopoly price maximises industry profits so that a higher price under vertical separation leads to lower industry profits than an integrated monopoly. Here, the mobile carrier's 
profits are $\frac{1}{16 b}\left(a-c_{F M}\right)^{2}$ while the fixed carrier's profits are $\frac{1}{32 b}\left(a-c_{F M}\right)^{2}$. Total profit equals $\frac{3}{32 b}\left(a-c_{F M}\right)^{2}$, less than the profit for an integrated monopoly given above.

Second, suppose that there are only two mobile networks. Solving the first order conditions for these two networks gives the termination charges as $T_{i}=\frac{1}{3 s_{i}}\left(a-c_{O}^{F}-c_{1}+\left(2 s_{i}-s_{j}\right) c_{T}^{M}\right)$ for each of the two mobile carriers $i$. The (average) price of fixed to mobile calls is given by $P=\frac{1}{6}\left(5 a+c_{F M}\right)>P^{m}$ with associated quantity $Q=\frac{1}{12 b}\left(a-c_{F M}\right)$. Note that in this situation that the price of fixed to mobile calls is higher than when there is a single mobile carrier. This is due to the effect of horizontal separation. Each network has an incentive to unilaterally raise its termination charge relative to the case of a single carrier as it gains the full price benefit of such a rise but shares any related loss in sales. This effect tends to be larger for small networks. Note that as the market share of any mobile carrier decreases, its (equilibrium) termination charge increases. In particular,

$$
\frac{\partial T_{i}}{\partial s_{i}}=-\frac{1}{3 s_{i}^{2}}\left(a-c_{F M}\right)<0 .
$$

At the same time, the share of the other carrier must increase and its termination charge falls. In the linear demand case considered here, the changes in termination charges that result from changes in mobile carrier market shares exactly offset each other. In other words, for the case of two mobile carriers, the price of fixed to mobile calls is independent of the shares of the individual mobile carriers even though the specific termination charges do depend on these shares.

The third case of interest allows for competition between an arbitrary number of symmetric mobile carriers. From the first order conditions, with $n$ mobile carriers, each of whom has a market share of $1 / n$, each carrier will independently set its termination charge in equilibrium at $T_{i}=\frac{n}{n+1}\left(a-c_{O}^{F}-c_{1}+\frac{1}{n} c_{T}^{M}\right)$. The price of fixed to mobile calls is $P=\frac{1}{2(n+1)}\left((2 n+1) a+c_{F M}\right)$. This price increases as the number of mobile carriers increase:

$$
\frac{d P}{d n}=\frac{1}{2(n+1)^{2}}\left(a+c_{F M}\right)>0 .
$$


For fixed to mobile calls there is not really any competition between mobile carriers. Because the person making the call is ignorant of the specific carrier they are calling, mobile carriers have no incentive to compete by offering a lower termination price. But as the number of carriers increases, the effect of horizontal separation rises and this pushes up the termination charges and the fixed to mobile price.

\subsection{Regulation}

There is clearly a range of regulatory rules that could be introduced by the authorities. We begin by considering two arms-length procedures then consider direct price regulation.

\subsubsection{Direct mobile charging}

As discussed in section 1, a minimal regulatory rule could involve the mobile carriers directly charging the A-party for call termination. The fixed carrier would only charge a customer the origination and trunk fee for a fixed-to-mobile call while the mobile carrier would charge the customer the termination charge. To make such a rule feasible, the actual billing would have to be done by the fixed carrier, possibly in return for a regulated billing fee.

Direct mobile charging does not alter customer ignorance. Customers still do not know which mobile network is associated with a specific number until after they have been billed. But it does alter the strategic interaction between the fixed and the mobile carriers.

To see the effects of direct mobile charging, suppose that the fixed network sets a price for origination and trunk carriage of $O$. The total price for a call from the fixed network to the mobile carrier $P_{i}=O+T_{i}$. Under customer ignorance, with the shares of each mobile company given by $s_{i}$, the expected price that the customer pays for a fixed to mobile call is $P=O+\sum_{i} s_{i} T_{i}$. The fixed carrier and mobile carriers simultaneously set their charges so that the fixed carrier solves:

$$
\max _{O} \frac{1}{2 b}\left(a-O-\sum_{i} s_{i} T_{i}\right)\left(O-c_{O}^{F}-c_{1}\right) .
$$

The first order condition for the fixed carrier is given by $a-2 O-\sum_{i} s_{i} T_{i}+c_{O}^{F}+c_{1}=0$. Each mobile carrier will solve: 


$$
\max _{T_{i}} \frac{s_{i}}{2 b}\left(a-O-\sum_{j} s_{j} T_{j}\right)\left(T_{i}-c_{T}^{M}\right) .
$$

The first order condition for each mobile carrier is $a-O-2 s_{i} T_{i}-\sum_{j \neq i} s_{j} T_{j}+s_{i} c_{T}^{M}=0$. Again, it is worth looking at three cases - a single mobile carrier, two mobile carriers with asymmetric market shares and $n$ symmetric mobile carriers.

First, if there is a single mobile carrier, solving the first order conditions gives $O=\frac{1}{3}\left(a-c_{T}^{M}+2 c_{O}^{F}+2 c_{1}\right)$ and $T=\frac{1}{3}\left(a-c_{O}^{F}-c_{1}+2 c_{T}^{M}\right)$. The total price of a fixed to mobile call is given by $P=\frac{1}{3}\left(2 a+c_{F M}\right)$. This price is below the standard double marginalisation price, as direct mobile charging has reduced the effect of vertical separation. At the same time, direct mobile charging introduces a new element of horizontal separation. The fixed and mobile components of the call are now complementary inputs from the consumers' perspective, but are provided by different firms. Thus, mobile direct charging does not completely internalise adverse spillovers in firm pricing and still leads to a price above the integrated monopoly price.

Second, suppose that there are two mobile carriers with potentially different market shares. In this situation,

$$
O=\frac{1}{4}\left(a+3 c_{O}^{F}+3 c_{1}-c_{T}^{M}\right) \text { and } T_{i}=\frac{1}{4 s_{i}}\left(a+c_{T}^{M}\left(3 s_{i}-s_{j}\right)-\left(c_{O}^{F}+c_{1}\right)\right) .
$$

The expected total price is $P=\frac{1}{4}\left(3 a+c_{F M}\right)$. As with the unregulated case, the total price of a fixed to mobile call is independent of the actual shares of the mobile carriers with linear demand. But the price is lower with direct mobile charging than with standard vertical separation. The specific termination charges set by each carrier will depend on their market shares and, as in the case without regulation, these termination charges tend to rise for a carrier as its market share falls.

Third, consider $n$ symmetric mobile carriers. Solving the first order conditions in this situation gives a total price of $P=\frac{1}{n+2}\left((n+1) a+c_{F M}\right)$. To see the effect of increasing the number of mobile carriers with direct charging,

$$
\frac{d P}{d n}=\frac{a-c_{F M}}{(n+2)^{2}}>0
$$

The price of fixed to mobile calls rises as the number of mobile carriers increases even with direct charging.

Overall, direct mobile charging, even in the presence of customer ignorance, tends to result in lower fixed to mobile prices than no regulation. But at the same 
time, it still leads to prices above the integrated monopoly level, and the price tends to rise as the number of mobile carriers increases.

\subsubsection{Carrier identification}

An alternative regulatory approach would involve carrier identification. At a minimum, this would involve advertising which carriers were associated with which numbers to reduce customer ignorance. Even so, this would only be a short-term solution and would not be amenable to mobile number portability. Carrier identification could involve a customer being informed of the identity of the mobile carrier that they are calling after they dial the number but before billing commenced. A simple sound may suffice. ${ }^{9}$ The customer would then have the option of terminating the call if they did not want to buy the relevant call.

If carrier identification perfectly removes customer ignorance then each mobile carrier is independent and there is no horizontal spillover between carriers. If there is no call substitution then the fixed carrier and each mobile carrier are, in effect, a separate vertical pair. In other words, if a customer wishes to call a person or company connected to a specific mobile carrier and there is no alternative to this call, then the fixed and relevant mobile carriers are just like sequential monopolists. As a result, the behaviour of the fixed carrier and each mobile carrier will be identical to the case analysed above for a single mobile carrier. In other words, for each mobile carrier, $T=\frac{1}{2}\left(a-c_{O}^{F}-c_{1}+c_{T}^{M}\right), P=\frac{1}{4}\left(3 a+c_{F M}\right)>P^{m}$ and $Q=\frac{s_{i}}{8 b}\left(a-c_{F M}\right)$. Carrier identification completely removes the effect of horizontal separation but it has no effect on vertical separation.

Alternatively, if there is call substitution between carriers, then each mobile carrier will have a reduced ability to exert any monopoly power. We would expect mobile termination charges to fall and this to be reflected in the fixed-to-mobile price. In the extreme, suppose that there is perfect substitution between calls to different mobile carriers. In this case, a customer has numerous firms or people that they could call, and these firms or people are connected to different networks. The customer does not care which firm or person they call and will simply choose the firm or person associated with the cheapest fixed-to-mobile price. This leads to perfect competition between the mobile carriers, and termination charges will be set at marginal cost. The 
fixed carrier, however, retains all of their monopoly power and can set the price of fixed-to-mobile calls equal to the monopoly price.

\subsubsection{Carrier identification and direct mobile charging}

It might seem sensible to use direct mobile charging to reduce the effect of vertical separation and carrier identification to remove the effect of horizontal separation. First consider the case with no call substitution between mobile networks. If these regulatory options are both implemented, then the result for the fixed network and each individual carrier will be like the situation of direct mobile charging with only one mobile carrier. In other words, for each mobile carrier, $O=\frac{1}{3}\left(a-c_{T}^{M}+2 c_{O}^{F}+2 c_{1}\right)$ and $T=\frac{1}{3}\left(a-c_{O}^{F}-c_{1}+2 c_{T}^{M}\right)$. The total price of a fixed to mobile call is given by $P=\frac{1}{3}\left(2 a+c_{F M}\right)$. Again it is worth pointing out that this price is below the price established with just carrier identification, but remains above the integrated monopoly price.

As substitution between mobile carriers increases, termination charges under direct mobile charging will decrease until, with perfect substitution, the charges equal marginal cost. The fixed carrier retains its monopoly power so that the origination charge will be set at the monopoly level, $O=P^{m}-c_{T}^{M}$.

\subsubsection{Direct setting of termination charges}

In contrast to the arms-length options considered above, the regulator could set termination charges directly. Suppose the regulator establishes a termination charge $\tau$ that must be set by all mobile carriers. The fixed carrier will then take this regulated charge as given and set the price of fixed to mobile calls to maximise profits. As the termination charges are now simply a cost to the fixed carrier, and all strategic interaction is removed, the profit maximising price of fixed to mobile calls will be $P=\frac{1}{2}\left(a+c_{O}^{F}+c_{1}+\tau\right)$. If we compare this with the integrated monopoly then $P-P^{m}=\frac{1}{2}\left(\tau-c_{T}^{M}\right)$. If the regulator sets the marginal termination charge equal to the marginal cost of termination, then the price will equal the integrated monopoly price. If the regulator sets a termination charge above marginal cost then the fixed-to-mobile

9 'AT\&T' have a simple identification sound in the US. 
price rises, while if the regulator sets a termination charge below the marginal termination cost, then the fixed-to-mobile price falls below the monopoly price.

The socially optimal price for fixed-to-mobile calls is given by the marginal cost of these calls, $c_{F M}$. The regulator would have to set the termination charge at $\tau^{*}=c_{O}^{F}+c_{1}+2 c_{T}^{M}-a$ before profit maximisation by the fixed carrier was aligned with the social optimum. ${ }^{10}$

For any higher termination charge, $\tau>\tau^{*}$, social welfare will be decreasing as the termination charge rises. In particular, note that it is not optimal for the regulator to set the termination charge equal to the marginal cost of termination. This only leads to integrated monopoly pricing and monopoly profits for the fixed carrier. Rather, to raise social welfare the regulator needs to set the termination charge below marginal cost. ${ }^{11}$ This creates a problem for the regulator. The mobile carriers will make a loss on terminating calls. This loss will need to be recouped from some other source. For example, if the regulator sets the termination charge below cost, but that is all, then the mobile carriers would have to recoup their loses from terminating calls by raising charges on outgoing calls. If competition prevented this, then some carriers would need to leave the industry to reduce competition and allow outgoing call prices to rise. In brief, setting the termination charges below cost to correct the distortion in fixed to mobile calls will lead to a reduction in mobile subscriber competition.

One alternative for the regulator is to set termination charges at zero. Such termination charges will help offset the monopoly pricing by the fixed carrier. If $a$ is large relative to $c_{F M}$, then a zero termination charge can still exceed $\tau^{*}$. But if $a$ is relatively close to $c_{F M}$ or $c_{T}^{M}$ is relatively large, then zero termination charges may be below the socially optimal level.

Zero termination charges have the advantage that they are easy to implement. The regulator simply does not allow mobile carriers to charge for termination. As the fixed carrier bills the customer, this carrier can enforce the rule by simply not passing any fees onto the mobile carriers. At the same time, zero termination fees will result in the mobile carriers making a loss from terminating fixed-to-mobile calls. To avoid

\footnotetext{
10 In practice the regulator will face standard information constraints when setting $\tau$. For example, the regulator may not know $c_{T}^{M}$. Baron and Myerson (1982) and Baron (1989) discuss this issue.
} 
these losses from impacting on other aspects of mobile phone competition, the mobile carriers will need to be compensated for this loss, say by a fixed per carrier transfer from the owner of the fixed network.

\subsubsection{Intervention for only a dominant carrier}

An alternative to direct price setting for all mobile carrier termination charges would be to just set the termination charge for a 'dominant' carrier. To see the effect of such a policy, suppose that there are $n$ mobile carriers, and they have asymmetric market shares. Let $s_{j}>s_{i}$ for all firms $i \neq j$ so that firm $j$ is the 'dominant' carrier. The regulator directly sets firm $j$ 's termination charge for fixed-to-mobile calls at $\tau$. All other carriers then simultaneously set their termination charges. Given these termination charges, the fixed carrier sets the price for fixed-to-mobile calls.

Using the first order conditions presented in section 2.2 above, replacing $T_{j}$ with $\tau$, we see that mobile carrier $i$ will set its termination charge so that

$$
a-\sum_{k \neq i, j} s_{k} T_{k}-s_{j} \tau-2 s_{i} T_{i}-c_{O}^{F}-c_{1}+s_{i} c_{T}^{M}=0 .
$$

To simplify, assume that all non-dominant (and non-regulated) mobile carriers are symmetric with market shares $s_{i}=\frac{1}{n-1}\left(1-s_{j}\right)$. Then, for each non-regulated mobile carrier,

$$
T_{i}=\frac{n-1}{n\left(1-s_{j}\right)}\left(a-c_{O}^{F}-c_{1}-s_{j} \tau+\frac{1-s_{j}}{n-1} c_{T}^{M}\right) .
$$

with

$$
P=\frac{1}{2 n}\left((2 n-1) a+c_{O}^{F}+c_{1}-s_{j} \tau+\left(1-s_{j}\right) c_{T}^{M}\right)
$$

Note that $P>P^{m}$ even if $\tau=c_{T}^{M}$ so long as $n \geq 2$. In other words, even if the regulator requires the dominant carrier to set its termination price at marginal cost, the resultant fixed-to-mobile price will always exceed the integrated monopoly price whenever there is at least one other mobile carrier.

This said, regulation of one mobile carrier leads to lower fixed-to-mobile prices than no regulation so long as the regulated price does not exceed the marginal

\footnotetext{
11 Armstrong, Cowan and Vickers (1996) discuss this issue for general access pricing with imperfect downstream
} 
termination cost by too much. To see this, let $\Delta P$ denote the difference between the fixed-to-mobile price with one mobile carrier regulated and the price with no regulation but the same total number of mobile carriers. Then $\Delta P=\frac{1}{2 n(n+1)}\left(-a+c_{O}^{F}+c_{1}+c_{T}^{M}+(n+1) s_{j}\left(\tau-c_{T}^{M}\right)\right)$. This will be negative so long as $\tau-c_{T}^{M}$ is not too positive.

The effect of dominant carrier regulation in reducing the equilibrium fixed to mobile price is small compared to the effect of additional mobile carriers in raising this price. To see this, suppose that one carrier is regulated but at the same time there is one more mobile carrier. In other words, while the dominant carrier is regulated, another unregulated mobile carrier enters the industry. Holding the market share of the dominant carrier fixed, let the change in price under regulation with an extra mobile firm compared to the case of no regulation with one less mobile firm be denoted by $\Delta \bar{P}$. Then $\Delta \bar{P}=\frac{1}{2(n+1)}\left(s_{j}\left(\tau-c_{T}^{M}\right)\right)$. This is positive if $\tau-c_{T}^{M}$ is positive.

\subsubsection{Regulation by setting the fixed-to-mobile price}

One final regulatory option should be noted. While the above analysis has considered the regulation of the termination charges set by the mobile carriers, an alternative solution to the problem of fixed-to-mobile charges might be the direct regulatory setting of the fixed-to-mobile call price. Setting the end price for fixed-tomobile calls avoids some of the problems with regulating mobile termination charges directly. In particular, it avoids issues of regulating firms that only have a relatively small market share. Once the final price is established, termination charges would then simply be a device used by the carriers to divide any profit that exists under the regulation. While the setting of these charges could lead to considerable dispute, such a dispute is more about sharing profits than about raising prices to the detriment of final customers.

\subsection{Integration}

In the discussion above, we assumed that each mobile carrier and the fixed carrier were independent. What if the fixed carrier also owns one of the mobile 
carriers? For simplicity, we refer to this as the case of integration. In this situation, the mobile carrier that is owned by the fixed carrier cannot effectively raise its marginal termination charge above marginal cost. Any such increase would merely transfer funds from the fixed carrier to the mobile carrier. But as the same company owns these carriers, the transfers would simply be internal to the company. A manager or owner who sought to maximise the total profits of the company would disregard such internal transfers as they have no effect on the profit of the company as a whole.

With integration, the fixed carrier will take the price of terminating calls to mobile phone networks other than its own as given, and will set the price of fixed-tomobile calls to maximise company profits. Let $s_{F}$ refer to the fraction of mobile phone customers who are subscribers to the mobile carrier that is owned by the fixed carrier. The integrated carrier will solve:

$$
\max _{P}\left(P-c_{O}^{F}-c_{1}-s_{F} c_{T}^{M}-\sum_{i \neq F} s_{i} T_{i}\right) Q(P)
$$

Fr linear demand, the solution to this problem is given by $P=\frac{1}{2}\left(a+s_{F} c_{T}^{M}+\sum_{i \neq F} s_{i} T_{i}+c_{O}^{F}+c_{1}\right) \quad$ with associated quantity $Q=\frac{1}{4 b}\left(a-s_{F} c_{T}^{M}-\sum_{i \neq F} s_{i} T_{i}-c_{O}^{F}-c_{1}\right)$. Given the behaviour of the fixed carrier, the non-integrated mobile networks will simultaneously set their termination charges to solve:

$$
\max _{T_{i}} \frac{s_{i}}{4 b}\left(T_{i}-c_{T}\right)\left(a-s_{F} c_{T}^{M}-\sum_{j \neq F} s_{j} T_{j}-c_{O}^{F}-c_{1}\right) .
$$

The first order condition for the mobile carriers optimisation is given by

$$
a-s_{F} c_{T}^{M}-\sum_{j \neq i, F} s_{j} T_{j}-2 s_{i} T_{i}-c_{O}^{F}-c_{1}+s_{i} c_{T}^{M}=0 .
$$

Solving these first order conditions simultaneously for all mobile carriers gives the Nash equilibrium termination charges.

The first order conditions for the fixed and non-integrated mobile carriers are mathematically identical to the case of no integration but direct regulation of a dominant mobile carrier with $\tau=c_{T}^{M}$. Consequently, the results with integration will be directly analogous to this situation. For example, if there are $n$ mobile carriers in total and each non-integrated carrier has an equal market share, then the termination price for the $(n-1)$ non-integrated carriers will be given by 


$$
T_{i}=\frac{n-1}{n\left(1-s_{F}\right)}\left(a-c_{O}^{F}-c_{1}-s_{F} c_{T}^{M}+\frac{1-s_{F}}{n-1} c_{T}^{M}\right)
$$

Given these termination charges the fixed-to-mobile price is given by:

$$
P=\frac{1}{2 n}\left((2 n-1) a+c_{O}^{F}+c_{1}-\left(2 s_{F}-1\right) c_{T}^{M}\right)
$$

Similarly, if there are only two mobile carriers, one integrated and one not integrated, then $T_{i}=\frac{1}{2}\left(a-c_{O}^{F}-c_{1}+c_{T}^{M}\right), P=\frac{1}{4}\left(3 a+c_{F M}\right)>P^{m}$ and $Q=\frac{1}{8 b}\left(a-c_{F M}\right)$.

From $\Delta \bar{P}$ above with $\tau=c_{T}^{M}$, integration together with entry by one extra nonintegrated mobile carrier leads to no change in the price of fixed-to-mobile calls. In this sense, integration of one mobile carrier with the fixed carrier is equivalent to 'losing' one mobile carrier in terms of the effect on price. For example, if there are only two carriers, one of which is integrated with the fixed carrier, then the price of fixed-to-mobile calls in equilibrium is the same as the 'double marginalisation' price with only one non-integrated carrier. In this case the non-integrated mobile carrier's profits are equal to $\frac{1}{16 b}\left(a-c_{F M}\right)^{2}$, the same as if it were the only mobile carrier. The integrated carrier makes profits $\frac{1}{32 b}\left(a-c_{F M}\right)^{2}$. These are the same as if it did not own a mobile carrier and there was only one non-integrated mobile carrier.

In this example, integration raises total industry profit but also 'diverts' profits to the non-integrated mobile carrier. This suggests that the fixed carrier might have no incentive to integrate. Or, if it is initially integrated, then the fixed carrier might have incentive to separate from its mobile carrier, particularly in the longer term when market shares of mobile carriers have 'settled down.' To confirm this, if there are $n$ symmetric non-integrated mobile carriers then the fixed carrier makes profit of $\Pi_{F}=\frac{1}{8 b(n+1)^{2}}\left(a-c_{F M}\right)^{2} \quad$ while each mobile carrier makes profit of $\Pi_{M}=\frac{1}{4 b(n+1)^{2}}\left(a-c_{F M}\right)^{2}$. In contrast, if the fixed carrier is integrated with one of the $n$ mobile carriers, then the integrated carrier makes total profits $\Pi_{I}=\frac{1}{8 b n^{2}}\left(a-c_{F M}\right)^{2}$. Integration will only be profitable if $\Pi_{I} \geq \Pi_{F}+\Pi_{M}$. Simplifying, it is only profitable to be an integrated carrier if $\frac{1}{8 b n^{2}}\left(a-c_{F M}\right)^{2} \geq \frac{3}{8 b(n+1)^{2}}\left(a-c_{F M}\right)^{2}$ or $2 n^{2}-2 n-1 \leq 0$. Solving this quadratic equation means that there is only an incentive to integrate if $n \leq 1.37$. 
To interpret this result, if all mobile carriers are symmetric, then integration will only be a stable long-run outcome if there is only one mobile carrier. The logic behind this is clear. Integration with one mobile carrier avoids double marginalisation and raises total profits. But for two or more mobile carriers, it is better not to integrate. In this case, the non-integrated mobile carriers seize all the benefits of integration, and integration actually makes the fixed carrier worse off.

There are two clear caveats to this result. First, if integration with the fixed carrier makes it easier to gain mobile customers and increase market share then integration is more likely to be desirable. This could occur through reputation or marketing. Secondly, if market shares are not symmetric then integration with the largest of the mobile carriers may be desirable even though there are two or more mobile carriers. This effect will tend to decrease as the number of mobile carriers rises and market shares become more equal over time.

\section{Mobile Network Competition}

The model presented in section 2 assumed that the market share of each mobile network was fixed and did not change as termination charges and fixed to mobile prices changed. In many situations, however, mobile networks compete with one another for subscribers. The costs and quality of their service critically influence this competition and to the extent that these are influenced by termination charges they are of relevance to this paper.

In this section we develop a model of mobile network competition in order to understand the determinants of the market shares of competing networks. Following Armstrong (1998) and Laffont, Rey and Tirole (1998a), we envisage negotiations or regulation of termination charges as occurring prior to the competition between mobile networks for customers. Consequently, we will take those charges as given as the model is developed. We will then consider how termination charges interact with mobile network competition.

For simplicity we concentrate on the case of two mobile networks, $A$ and $B$, and a single fixed line network, $F$. The fixed network has a set of customers of measure $\alpha$ while the mobile networks compete for a customer set of measure 1 . The value of $\alpha$ can be interpreted as a measure of the relative size of the fixed customer 
base to the mobile base. We assume that these overall shares are fixed: in effect, fixed and mobile customers are distinct and the two forms of telecommunication are not competitors. ${ }^{12}$

We maintain our notation from the previous section but add some new definitions. In particular,

- $c_{T}^{F}$ is the marginal cost of termination on the fixed network

- $c_{O}^{M}$ is the marginal cost of origination on either mobile network

- $\quad c_{1}$ is the marginal trunk cost incurred by fixed and mobile networks for calls to each other

- $f$ is the cost of connecting a consumer to a mobile network

Thus, mobile and fixed networks can potentially have different cost structures.

The two mobile networks, $A$ and $B$, sell differentiated but substitutable products. We model this by assuming that each network is located at either end of a line of length 1 with $A$ located at 0 and $B$ located at 1 . Consumers are located uniformly over the line. Given income $y$, outgoing calls $q$ and incoming calls $z$, a consumer located at $x$ and joining network $i$ has utility:

$$
y+v_{0}-t\left|x-x_{i}\right|+u(q, z)
$$

where $v_{0}$ represents a consumer's intrinsic value of having a mobile phone and $t\left|x-x_{i}\right|$ denotes the cost of joining a network with 'address' $x_{i}(i=A, B)$ that differs from the consumer's own location. ${ }^{13} u(q, z)$ captures the consumer's utility from making and receiving calls. To simplify the analysis we initially assume that the consumer is indifferent to incoming calls so that $u(q, z)=u(q)$. Consequently, the price of fixed-to-mobile calls will not directly concern the mobile subscriber, although, as we show below, these prices have significant indirect effects through subscriber competition. We comment on the implications of consumer preferences for incoming calls later in this section.

\footnotetext{
12 We will consider the implications of relaxing this assumption towards the end of this section.

13 This is essentially the model structure of Laffont, Rey and Tirole (1998a, 1998b).
} 


\subsection{Pricing and the Determination of Market Shares}

We can now consider a consumer's choice between mobile carriers. Let $v(p)$ be the indirect utility function of a consumer who faces an outgoing call price of $p$ (per call or call-minute) (i.e., $v(p) \equiv \max _{q} u(q)-p q$ ). We interpret this, as do Laffont, Rey and Tirole (1998b), as the utility generated from calling a particular person. Implicitly, this means that consumers do not refrain from calling people when the price of doing so rises but merely that they call them for less often or for smaller periods of time.

We will assume that mobile consumers can be offered non-linear prices, i.e., a two-part tariff. That is,

$$
W_{i}\left(q_{i}, q_{i j}, q_{i F}\right)=F_{i}+p_{i} q_{i}+p_{i j} q_{i j}+p_{i F} q_{i F}
$$

where:

- $F_{i}$ is the fixed fee or subscriber charge;

- $\quad p_{i}$ is the per call intra-network charge on network $i$;

- $q_{i}$ is the quantity of calls on network $i$;

- $\quad p_{i j}$ is the per call charge from network $i$ to network $j$

- $\quad q_{i j}$ is the quantity of calls from network $i$ to network $j$

- $\quad p_{i F}$ is the per call charge from network $i$ to network $F$

- $q_{i F}$ is the quantity of calls from network $i$ to network $F$

Further, let $T_{i j}$ be the termination charge from network $i$ to network $j$ and $T_{i F}$ be the termination charge for the fixed network. $p_{F i}, T_{F i}$ and $q_{F i}$ are defined analogously. Consider an 'average' consumer whose (expected) callng pattern matches the distribution of customers over the fixed and mobile networks. Then the call utility for that consumer from subscribing to either network $A$ or $B$ is:

$$
\begin{aligned}
& V_{A}=\frac{1}{\alpha+1}\left(s v\left(p_{A}\right)+(1-s) v\left(p_{A B}\right)\right)+\frac{\alpha}{\alpha+1} v\left(p_{A F}\right)-F_{A} \\
& V_{B}=\frac{1}{\alpha+1}\left(s v\left(p_{B A}\right)+(1-s) v\left(p_{B}\right)\right)+\frac{\alpha}{\alpha+1} v\left(p_{B F}\right)-F_{B}
\end{aligned}
$$


where $s$ is the market share (among mobile consumers) of network $A .{ }^{14}$

The market share of network $A, s$, is determined by the point of indifference between $A$ and $B$. That is,

$$
\begin{aligned}
& V_{A}-t s=V_{B}-t(1-s) \\
& \Rightarrow s=\frac{1}{2}+\sigma\left(V_{A}-V_{B}\right)
\end{aligned}
$$

where $\sigma=1 /(2 t)$ is the degree of substitutability between the two mobile networks.

Note that in bidding for customers, each network will find it optimal to set each call charge equal to its marginal cost. Focusing on network $A$, these usage prices are: $p_{A}=c_{1}+c_{O}^{M}+c_{T}^{M}, p_{A B}=c_{1}+c_{O}^{M}+T_{B A}$, and $p_{A F}=c_{1}+c_{O}^{M}+T_{F A}$. This is a common outcome when firms can set multi-part tariffs. It is in a network's interest to set nondistortionary tariffs, because when price is set equal to marginal cost, the sum of consumer surplus and that network's per customer profits is maximised. If this were the only component of pricing, the network may not recover any fixed costs, e.g., customer connection costs. However, the fixed charge, $F_{A}$, can be utilised to divide total value created (that is, utility less costs). ${ }^{15}$

Because our focus is on fixed-to-mobile prices we will fix the other termination charges at their respective marginal termination costs. That is, $T_{A B}=T_{B A}=c_{T}^{M}$ and $T_{F A}=T_{F B}=c_{T}^{F}$. These are the outcomes that would result from efficient bargaining between those carriers (Laffont, Rey and Tirole, 1998a).

Given these simplifying assumptions, the market share of network $A$ becomes:

$$
s=\frac{1}{2}+\sigma\left(F_{B}-F_{A}\right)
$$

Note that an interior equilibrium exists if $\sigma$ (the degree of mobile network substitution) is not too high.

\subsection{Equilibrium Mobile Charges}

We can now consider the mobile networks' choice of subscription charge, $F_{A}$ and $F_{B}$, given the fixed-to-mobile termination charges $T_{A F}$ and $T_{B F}$. Each network will choose their charge to maximise its profits taking the other network's prices as

\footnotetext{
14 Effectively, this is an expected utility calculation reflecting the utility of calling a specific person whose probability of belonging to a network is given by that network's market share.

${ }^{15}$ For a discussion of this see King and Maddock (1996).
} 
given. In a Nash equilibrium (if it exists), each network will be satisfied with their choices and find unilateral deviations unprofitable.

Given our assumptions network $A$ 's profit is,

$$
\pi_{A}=s\left(F_{A}-f\right)+\frac{\alpha}{\alpha+1} s\left(T_{A F}-c_{T}^{M}\right) Q .
$$

where $q_{F A}=Q$ under customer ignorance. This profit is the sum of the mobile network's profits from its own customers and the profit from terminating calls from the fixed network. $A$ will choose $F_{A}$ to maximise these profits, yielding the first order condition:

$$
\frac{\partial \pi_{A}}{\partial F_{A}}=\frac{1}{2}+\sigma\left(F_{B}-F_{A}\right)-\sigma\left(F_{A}-f+\frac{\alpha}{\alpha+1}\left(T_{A F}-c_{T}^{M}\right) Q\right)=0
$$

Raising its charge increases the direct revenues $A$ earns from its inframarginal customers. However, it also drives away marginal customers. This is costly because not only does $A$ lose its direct revenues but also the termination revenues it would otherwise receive from customers on the fixed network calling those marginal customers. $B$ faces the same trade-off when determining its subscription charge.

It is important to note that the existence of fixed-to-mobile termination profits makes networks tougher price competitors for subscribers. When networks discount to gain a customer, they also attract the calls from the fixed network to that customer. This makes the marginal customer more valuable and hence, bidding for that customer more intense. To see this, note that given $T_{A F}$ and $T_{B F}$, per subscriber termination profits are $\tilde{T}_{A}=\frac{\alpha}{\alpha+1}\left(T_{A F}-c_{T}^{M}\right) Q$ and $\frac{\partial^{2} \pi_{A}}{\partial F_{A} \partial \tilde{T}_{A}}=-\sigma \leq 0$.

The fixed line network also chooses its prices following the setting of termination charges. With linear demand, its first order condition is as before:

$$
\frac{1}{2 b}(a-P)+\left(c_{1}+c_{O}^{F}+s T_{A F}+(1-s) T_{B F}\right) \frac{1}{2 b}=0
$$

Simultaneously solving the three first order conditions, we find that:

$$
F_{A}=\frac{\alpha \sigma(3+2 f \sigma) T_{A F}^{2}-3 \alpha \sigma c_{T}^{M}\left(2\left(a+c_{1}+c_{O}^{F}\right)+T_{A F}+T_{B F}\right)+\alpha \sigma\left(4\left(a+c_{1}+c_{O}^{F}\right)+T_{B F}(1-4 f \sigma)\right)+2\left(\alpha \sigma\left(a+c_{1}+c_{O}^{F}\right) T_{B F}-6 b(1+\alpha)(1+2 f \sigma)+\alpha \sigma T_{B F}^{2}\right)}{2 \sigma\left(\alpha \sigma\left(T_{A F}-T_{B F}\right)^{2}-12 b(1+\alpha)\right)}
$$


So long as $\sigma$ is low enough (i.e., mobile networks are relatively poor substitutes), then this is the unique (Nash) equilibrium. Perhaps more instructive is the equilibrium market share for network $A$ :

$$
s=\frac{1}{2}+\frac{\sigma \alpha}{3(1+\alpha)}\left(T_{A F}-T_{B F}\right) Q
$$

Notice that when $T_{A F}=T_{B F}, s$ is equal to $1 / 2$. When $T_{A F}>T_{B F}$ results in $s>\frac{1}{2}$ as this raises the relative revenue $A$ earns from its customers and hence, increases the attractiveness of competing for those customers.

\subsection{Fixed-to-Mobile Prices}

As for the case of fixed market shares, the analysis of fixed-to-mobile prices and mobile network termination charges depends critically upon how informed consumers are regarding the network they are calling. If they cannot distinguish between alternative networks they will base their demand on a weighted price and the outcome will be as if the fixed network charged the same price for calls to each mobile network. On the other hand, if they can make such distinctions, price discrimination is possible.

We continue to assume consumer ignorance. This means that $q_{F A}=s Q$ and $q_{F B}=(1-s) Q$. Recall from the fixed market share case that the price of fixed to mobile calls, $P$, is a monopoly price, $P=\frac{1}{2}\left(a+s T_{A F}+(1-s) T_{B F}+c_{O}^{F}+c_{1}^{F}\right)$. Given the equilibrium market shares, as defined by (5.5), the total quantity of fixed to mobile calls is:

$$
Q=\frac{3(1+\alpha)\left(a-c_{O}^{F}-c_{1}^{F}-\frac{1}{2}\left(T_{A F}+T_{B F}\right)\right)}{12 b(1+\alpha)+\alpha \sigma\left(T_{A F}-T_{B F}\right)^{2}}
$$

The profit equation for network $A$ (taking into account the interior equilibrium in competition for mobile customers) is:

$$
\pi_{A}=\frac{1}{4 \sigma}\left(1+\frac{2 \alpha}{3(1+\alpha)} \sigma Q\left(T_{A F}-T_{B F}\right)\right)^{2}
$$

$A$ chooses $T_{A F}$ to maximise these profits. The first order condition is:

$$
Q+\left(T_{A F}-T_{B F}\right) \frac{\partial Q}{\partial T_{A F}}=0
$$


Essentially, the mobile networks act as relative profit maximisers on their termination charges. The intuition here is that own profit is passed on to mobile subscribers through price competition unless termination profits exceeds that of the other mobile network. If it does, then the difference can be appropriated. Consequently, mobile networks can maximise total profit by ensuring that their termination profits are high relative to the other network.

Consider a symmetric equilibrium where $T_{A F}=T_{B F}$. It is easy to see that in that equilibrium $Q=0$ or $T_{A F}=T_{B F}=a-c_{O}^{F}-c_{1}^{F}$. That is, mobile termination charges are set so high that demand for fixed to mobile calls reaches its 'choke point' as defined by $P=a$. The intuition here is simple. Suppose $P=a$ and $Q=0$. Network $A$ could improve its own termination profits by setting its termination charge below $a-c_{O}^{F}-c_{1}^{F}$. However, this will confer a greater increment to termination profits of the rival network. This is because that network enjoys the benefit of a positive number of fixed to mobile calls but a greater mark-up for those units. Consequently, while termination profit may increase, relative termination profits and hence, overall profits falls.

\subsection{Preferences for Incoming Calls}

The analysis above shows that when mobile termination charges are set independently, they tend to be above marginal cost, and in the case of termination charges for fixed calls, they may be set so high as to 'choke' off fixed to mobile demand. One assumption in our model was that mobile consumers do not care about the quantity and hence, price of incoming calls from other networks. It is easy to see, however, that given our assumption of customer ignorance, consumer preferences for incoming calls do not alter our results.

Suppose that mobile consumers cared about the quantity of calls they received from the fixed line network. ${ }^{16}$ When evaluating which network to choose they will look at more than simply the subscription charge they face. They will also consider the fixed to mobile price charged to consumers and the quantity of calls received by the network. However, under customer ignorance, fixed to mobile prices do not

16 Also, suppose the consumers were not 'large' in the sense that they commanded monopsony power. 
effectively differ among networks. Therefore, regardless of which network they join, the quantity of incoming calls will be the same. It is only when fixed-line customers are aware of the network that they are calling and the price of that network that consumer preferences for incoming calls will become salient and impact upon termination prices.

\subsection{Regulation}

In general, to determine the quantitative effects of structural regulatory rules such as carrier identification or direct mobile charging, we need to resolve the entire model presented above. However, for carrier identification we can easily determine the qualitative effects of regulation.

When fixed line consumers can distinguish between the mobile networks they are calling and have knowledge of any price differentials, the termination charges and fixed to mobile call prices are the same as the fixed market share case. In this case, there is no horizontal externality from changing termination charges. Market shares from subscriber competition still depend on relative termination profits, but equilibrium will no longer involve choking-off fixed-to-mobile calls.

To see this, suppose that $T_{B F} \geq a-c_{0}^{F}-c_{1}$. No calls will be made from the fixed network to network $B$ regardless of $T_{A F}$ and network $B$ will make no termination profits. Clearly, network $A$ 's best response is to set $T_{A F}<a-c_{0}^{F}-c_{1}$. This will raise both $A$ 's relative and absolute termination profits. ${ }^{17}$ Thus, $A$ will gain a bigger subscriber base and make greater total profit. It immediately follows that there cannot be an equilibrium where both networks set termination charges to choke fixedto-mobile demand. Carrier identification will lower the average price of fixed to mobile calls. ${ }^{18}$ Note also that mobile subscribers will not face higher fees and may face lower fees in equilibrium with carrier identification. For example, if there is a

\footnotetext{
17 The key difference is that under customer ignorance, lowering $T_{A F}$ below the choke price raised A's absolute termination profits but lowered its relative termination profits so that total profits fell, not rose.

18 Formally, under carrier identification $\pi_{A}=\frac{1}{4 \sigma}\left(1+2 \sigma \frac{\alpha\left(\left(T_{A F}-c_{T}^{M}\right) q_{F A}-\left(T_{B F}-c_{T}^{M}\right) q_{F B}\right)}{3(1+\alpha)}\right)^{2}$. Noting that $q_{F A}$ is decreasing in $T_{A F}$, if $q_{F B}=0$ then $\pi_{A}$ is maximised when $q_{F A}$ is positive and $T_{A F}$ is strictly between the choke price and $c_{T}^{M}$.
} 
symmetric equilibrium both mobile networks will make positive termination profits and this will be 'passed back' to mobile consumers through subscriber competition. Thus carrier identification benefits both mobile and fixed network customers.

The direct setting of termination charges by a regulator involves a similar general benefit to customers in our framework. If a regulator sets termination charges for all mobile carriers below the choke price then in any symmetric equilibrium all customers gain. This said, it is important to understand that the driving force behind these gains is the reduction in the horizontal externality that exists between unregulated mobile networks under customer ignorance. Because of ignorance over the identity of the terminating mobile network, fixed line customers reduce the quantity of fixed-to-mobile calls that they buy when one mobile carrier raises its termination charges, but they do not alter their distribution of calls between mobile networks. Further, mobile network profit from subscriber competition depends on relative termination profits as each mobile carrier uses these profits to induce subscribers to join their network. It pays each mobile carrier to always raise its relative termination charge because this lowers the other carrier's termination profits and raises their own relative termination profits. ${ }^{19}$ This leads to a race between the mobile carriers to raise fixed-call termination charges to the choke price. Both carrier identification and direct regulatory price setting short-circuit this race. Carrier identification achieves this by eliminating the horizontal externality in termination profits. Direct regulation, in contrast, simply makes the race illegal.

\section{$4 \quad$ Future Directions}

This paper has analysed the determinants of termination charges for calls to mobile networks. Because of the number of prices involved and the potential complexity of strategic interactions we have kept the assumptions relatively minimal and symmetric. In particular, we have assumed considerable knowledge of consumer demand, simple horizontal product differentiation, and equivalent mobile network technologies. Nonetheless, we believe that such assumptions are not restrictive and relaxing them would not alter our key results. 
That said, there are issues raised by our research that are still unresolved. First, our results clearly depend on the assumption that mobile networks set a uniform termination price for fixed line calls. But efficient termination pricing is possible and can be facilitated by non-linear pricing such as two-part tariffs. While we know that usage charges in such pricing schemes should reflect marginal costs the issue of fixed charges is more difficult. Current research suggests that these should be structured to induce optimal investment incentives. ${ }^{20}$ However, the analysis here was essentially static and, as such, not able to address such issues.

Indeed, introducing dynamic analysis into the model would allow other issues to be addressed. For example, we have assumed that mobile users and fixed line users are independent. This neglects the important issue of the adoption of mobile services by consumers and their interaction with consumers who also consume fixed line services. It also neglects the role for new wireless technologies and their impact on mobile competition.

Similarly, our model did not consider entry and its effects. In particular, we did not consider explicitly the role of termination charging in altering the incentives of entrants. This too remains a fruitful area for future research.

In concluding, it should be noted that our analysis here is not at all specific to mobile network issues. Indeed, to the extent that consumers have difficulty distinguishing among competing carriers (a problem exacerbated by the steady introduction of local number portability), then these considerations would apply to interactions between many telecommunications networks including fixed line networks and data communication services. Thus, we expect that the results on termination presented here would also apply to many other inter-network regulatory situations in telecommunications.

\footnotetext{
19 Consequently, so long as termination charges are above their 'monopoly' level, lowering those charges will result in a reduction in mobile subscription charges.

${ }^{20}$ See Gans and Williams (1999) and Gans (1999) for a discussion of regulated pricing and investment incentives.
} 


\section{References}

ACCC (1999), "Principles for determining access prices for Domestic GSM Terminating Access and Domestic GSM Originating Access services," Discussion Paper, Melbourne.

Armstrong, M. (1997), "Competition in Telecommunications," Oxford Review of Economic Policy, 13 (1), pp.64-81.

Armstrong, M. (1998), "Network Interconnection in Telecommunications," Economic Journal, 108 (May), pp.545-564.

Armstrong, M., S. Cowan and J. Vickers (1996), "The Access Pricing Problem: A Synthesis," Journal of Industrial Economics, 44 (2), pp.131-150.

Baron, D. (1989), "Design of Regulatory Mechanisms and Institutions," R. Schmalensee and R.D. Willig (eds.), Handbook of Industrial Organization, Volume 2, Amsterdam: North-Holland, pp.1347-1447.

Baron, D. and R. Myerson (1982), "Regulating a Monopolist with Unknown Costs," Econometrica, 50, pp.911-930.

Carter, M. and J. Wright (1999), "Interconnection in Network Industries," Review of Industrial Organization, 14 (1), pp.1-25.

Doyle, C. and J.C. Smith (1998), "Market Structure in Mobile Telecoms: Qualified Indirect Access and the Receiver Pays Principle," Information Economics and Policy, 10 (4), pp.471-489.

Gans, J.S. (1998), "Regulating Private Infrastructure Investment: Optimal Pricing of Access to Essential Facilities," Working Paper, No.98-13, Melbourne Business School.

Gans, J.S. and S.P. King (1999), "Regulation of Termination Charges for NonDominant Networks," Working Paper, No.99-20, Melbourne Business School.

Gans, J.S. and P.L. Williams (1999a), "Access Regulation and the Timing of Infrastructure Investment," Economic Record, 79 (229), pp.127-138.

King, S.P. and R. Maddock (1996), Unlocking the Infrastructure, Sydney: Allen \& Unwin.

King, S.P. and R. Maddock (1999), "Light-handed Regulation of Access in Australia: Negotiation with Arbitration," Information Economics and Policy, 11 (1), pp.1-22.

Laffont, J-J., P. Rey and J. Tirole (1998a), "Network Competition I: Overview and Nondiscriminatory Pricing," RAND Journal of Economics, 29 (1), pp.1-37. 
Laffont, J-J., P. Rey and J. Tirole (1998b), "Network Competition II: Price Discrimination," RAND Journal of Economics, 29 (1), pp.38-56.

Monopolies and Merger Commission and Oftel (1998), Cellnet and Vodafone, London.

Perry, M. (1989), "Vertical Integration: Determinants and Effects," R. Schmalensee and R.D. Willig (eds.), Handbook of Industrial Organization, Volume 1, Amsterdam: North-Holland, pp.183-258. 


\section{University Library}

\section{- M M I E R R A A gateway to Melbourne's research publications}

Minerva Access is the Institutional Repository of The University of Melbourne

\section{Author/s:}

Gans, Joshua S.;King, Stephen P.

Title:

Mobile Network Competition, Customer Ignorance and Fixed-to-Mobile Call Prices

Date:

2000-02

Citation:

Gans, Joshua S. and King, Stephen P. (2000) Mobile Network Competition, Customer Ignorance and Fixed-to-Mobile Call Prices.

Persistent Link:

http://hdl.handle.net/11343/33664 